\title{
EVALUATION OF FIBER ORIENTATION OF AC AND DC ELECTROSPUN PLCL NANOFIBROUS LAYERS
}

\author{
${ }^{1}$ Nikifor ASATIANI, ${ }^{1}$ Jan VALTERA, ${ }^{1}$ Maxim LISNENKO, ${ }^{1}$ Kristyna HAVLICKOVA, \\ ${ }^{1}$ Jan KOVACICIN, ${ }^{1}$ David LUKAS, ${ }^{1} E v a$ KUZELOVA KOSTAKOVA, ${ }^{2}$ Jiří MOLÁČEK \\ ${ }^{1}$ Technical University of Liberec, Liberec, Czech Republic, EU, nikifor.asatiani@tul.cz, eva.kostakova@tul.cz \\ ${ }^{2}$ Faculty of Medicine in Pilsen, Charles University, Prague, Czech Republic, EU
}

https://doi.org/10.37904/nanocon.2021.4353

\begin{abstract}
Electrospun PLCL nanofibrous materials produced by direct current (DC) or alternating current (AC) electrospinning are good candidates for absorbable reinforcement for cardiovascular surgery composite bandages. For an appropriate description of the fiber layers and prediction of its relevant biomechanical characteristics, the knowledge of fiber orientation in the electrospun layers is essential. In this study, the different orientation of fibres in $A C$ and $D C$ electrospun materials was achieved only by changing the rewinding speed of the supporting material, as the easy way for the production of relatively large samples with controlled fiber orientation. The fiber orientation was measured on scanning electron microscope images at different magnification using an open-source image processing software $\mathrm{FIJI} / \mathrm{Image} \mathrm{J}$ with the implementation of the "OrientationJ" plugin. Subsequently, the fiber layers were compared in terms of fiber orientation. Optimal image parameters for the measurement and the best unidirectional oriented-aligned samples were determined
\end{abstract}

Keywords: Electrospinning, biodegradable nanofibers, aligned nanofibers, image analysis, scanning electron microscopy

\section{INTRODUCTION}

Electrospun fibrous materials are widely used in medical applications nowadays [1,2]. One of the important applications of nanofibrous materials are scaffolds for cardiovascular systems. The different electrospun nanofibrous structures are used. Thus, outer circumferentially aligned electrospun nanofibers are also used as a parts of small-diameter vascular grafts [3] or bandages, which application is based on the mechanical interaction of the arterial wall and the external support. The electrospun nano or microfibrous material morphological testing is necessary. However, not only fiber diameter distribution is essential to observe. It is necessary to check also fiber orientation. The fiber orientation or alignment of fibers in electrospun layer can dramatically influence mainly mechanical properties but also biological properties such as cell orientation, adhesion, migration etc. In order to evaluate the suitability of electrospun materials, it is necessary not only morphological analyses and spinnability analyses, etc., but it is also necessary to look at the orientation of the fibres in the materials produced, and this paper deals with this.

Today, both a high-voltage direct current (DC) source and an alternating current (AC) source are used to form polymer nanofibers. The use of a DC source requires an electrically active collector, but the AC source does not. The nature of both processes also indicates differences in the resulting morphologies of nanofiber layers, even in terms of fiber orientation controlled only by the rate of withdrawal of the base material. The orientation of fibres in DC electrospun materials with the special collectors using was studied several times for medical applications mainly [4-8]. The difference between the two technologies with changes in towing speed is described in this paper. The selection of a suitable magnification of images from a scanning electron microscope is also taken into account and the optimal procedure for evaluating such images for their 
comparison is set. This study shows how these electrospinning technologies used for the industrial production of nanofibrous materials find it difficult or almost unintentional to change the orientation of the fibers during the electrospinning process.

\section{MATERIALS AND METHODS}

\subsection{Fabrication of nano-/microfibrous layers}

A copolymer of L-lactide and $\varepsilon$-Caprolactone in a 70/30 molar ratio and with an inherent viscosity midpoint of $1.5 \mathrm{dl} / \mathrm{g}$ (Purasobr PLCL 7015, Corbion) was used for fabrication of the materials. The PLCL copolymer is biocompatible, biodegradable, and has very good mechanical properties for the potential application in cardiovascular surgery as a vascular biodegradable bandage. For the DC and AC electrospinning, the polymer solution was prepared with solvent system acetic acid/formic acid/acetone in weight ratio 1:1:1. The final polymer concentration was $10 \mathrm{wt} \%$. All the materials were electrospun from the freshly prepared solution after 4 hours at the magnetic stirrer. The electrospinning was done by DC needleless electrospinning by Nanospider ${ }^{\mathrm{TM}}$ machine, where static wire spinning electrode is used. The AC device with a cylindrical spinning electrode and a rotating cylinder as an electrically inactive collector was applied to create a second set of electrospun materials. The schematic difference in between these two electrospinning technologies is introduced in Figure 1.
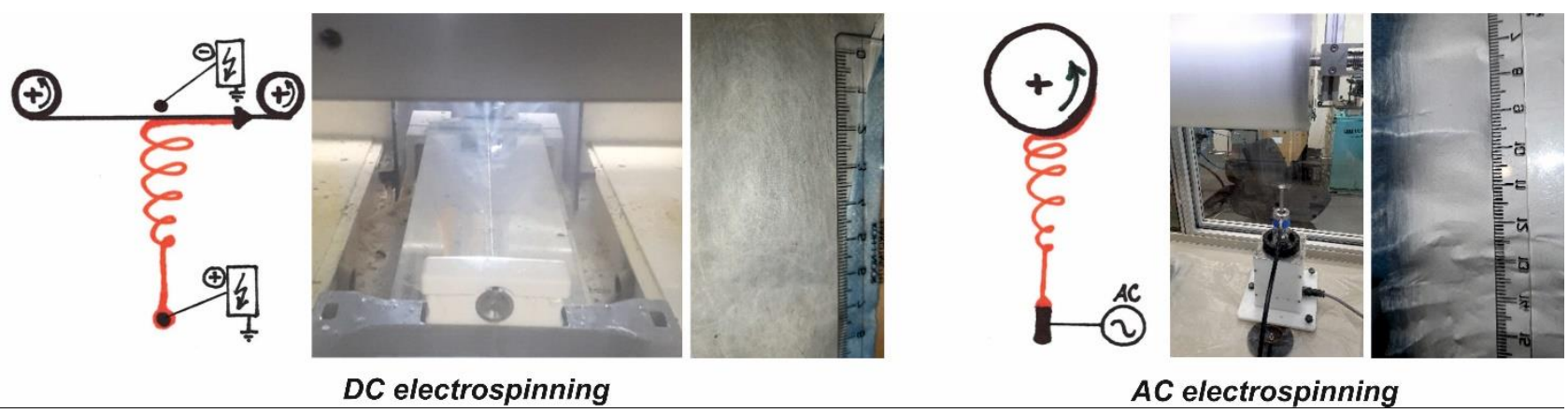

Figure 1 Scheme and photos of used DC and AC electrospinning devices and examples of electrospun nanofibrous layers on the nonwoven supporting materials

All the nanofibrous materials were DC electrospun on a needless electrospinning device type NS 1WS500U (Elmarco, CZ) based on a stationary wire spinning electrode. The spinning electrode was charged with direct current high voltage source positively $(40 \mathrm{kV})$, and the collector was charged negatively $(-10 \mathrm{kV})$. The distance between the spinning electrode and the collector was $180 \mathrm{~mm}$. The polymer solution was fed onto stationary wire spinning electrode by moving carriage module filled with a polymer solution. Metal insert orifice $0.7 \mathrm{~mm}$ in diameter was used for control of polymer solution layer on the spinning electrode. The DC electrospinning device is complemented by an air conditioning unit and thanks to that the exact values of temperature and air humidity in the spinning space were used. The temperature was $22^{\circ} \mathrm{C}$ and the relative humidity was $40 \%$. The used supporting material was spunbond Pegatex S (PFNonwovens, CZ) with $20 \mathrm{~g} / \mathrm{m}^{2}$ and the withdrawal speeds were changed according to Table 1.

The second set of samples was prepared on a device using a high voltage AC source. This device was designed and constructed at the Technical University of Liberec $[9,10]$. The distance between the spinning electrode and the collector was $180 \mathrm{~mm}$. The perforated rotating cylinder with $30 \mathrm{~cm}$ in diameter coated with a supporting spunbond nonwoven Pegatex S (PFNonwovens, CZ) with $20 \mathrm{~g} / \mathrm{m}^{2}$ was used as an electrically inactive collector capturing the formed nanofibers. The withdrawal speed introduced in Table 1 represents the circumferential speed of rotation of the cylinder collector. The value of the effective electric voltage was $35 \mathrm{kV}$. The device is not equipped with an air conditioning unit, but the ambient conditions were measured. The 
temperature during the $\mathrm{AC}$ electrospinning was $21^{\circ} \mathrm{C}$, and the relative humidity was $43 \%$. The minimal peripheral speed of rotating perforated cylinder as electrically inactive collector was $30 \mathrm{~mm} / \mathrm{min}$, slower speed does not allow sufficient catching of fibres on the roller surface. The maximal peripheral speed was $100 \mathrm{~mm} / \mathrm{min}$. When the higher speed was used, the fibres broke. The nanofibrous materials immediately removed from the cylindrical collector after spinning showed large precipitation along the length. For this reason, the sample was also tested and the sample was left on the cylinder for 24 hours to dry the residual solvents completely and to relax the stress in the sample.

Table 1 Withdrawal speeds set up for DC and AC electrospinning to produce nanofibrous layer

\begin{tabular}{|l|c|c|c|c|c|}
\hline & \multicolumn{5}{|c|}{ Withdrawal speed of supporting nonwoven material [mm/min] } \\
\hline DC electrospinning & 0 & 6 & 20 & 100 & 215 \\
\hline AC electrospinning & 30 & 80 & 80 (left on roller for 24 hours) & 100 & \\
\hline
\end{tabular}

\subsection{Characterization of produced fibres}

Scanning electron microscopy (SEM) was used to analyse the alignments of obtained fibrous layers using Orientation J Distribution plugin in the FIJI/ImageJ software $(\mathrm{NIH})$. Each submitted SEM image was first deframed so that the entire field of view could be considered the area of interest. Some of the images have been rotated $90^{\circ}$ to make the histograms easier to read. In particular, the methodology of measuring several selected regions of interest on one image was also tested. If necessary, contrast and sharpness of an image were adjusted. Before each measurement, it was important to set three parameters -1 ) sigma, $\sigma$, or a rough estimate of the size of the structure of interest (i.e., the fiber diameter in pixels); 2) the desired coherence (i.e., uniformity or continuity) and energy (this parameter allow to "distinguish" fiber from the background), \%. The resulting fiber orientation is then judged on the basis of a color-coded map, where hue corresponds to the angle of local fiber orientation and saturations reflect the coherence. The angle of local orientation can range from $-90^{\circ}$ to $90^{\circ}$ relative to the horizontal. Supplementary video accompanies this paper at https://youtu.be/u7b1 uKlo0M.

\section{RESULTS AND DISCUSSION}

Using the OrientationJ plugin, images of nanofibrous layers fabricated with different withdrawal speeds were analysed. The main focus was on the feasibility and suitability of using the plugin to obtain qualitative and quantitative information to assess fiber orientations based simply on SEM images. Images at different magnifications and their influence on the resulting measurements were tested. The effect of image threshold settings, contrast and sharpness of individual images was also investigated. Finally, different input parameters such as coherence, energy and sigma were tested. It was experimentally discovered that $10 \%$ energy and $10 \%$ coherence are optimal for most of the SEM images as input values. The most sensitive parameter of the measurement, however, is $\sigma$, or a rough estimate of the size of the structure of interest. As this parameter corresponds to the size of individual fibres, the correct estimation of $\sigma$ before the measurement was crucial. The essential problem with an estimation of $\sigma$ can occur when the fibrous layer has a multimodal distribution of fiber diameters.

The analyzed SEM images of DC electrospun fibrous layers revealed that it was nearly impossible to control the fiber orientation with rewinding speed of the substrate. It can be seen from Figure 2 that even at high speed ( 100 or $215 \mathrm{~mm} / \mathrm{min}$ ) it was difficult to collect fibers in a controlled manner. The main difference between the materials fabricated at low (6 or $20 \mathrm{~mm} / \mathrm{min}$ ) or even zero speed was in the thickness and density of the obtained layers. As Figure 2 indicates, the high density can negatively affect the orientation measurement. With increasing density of fibers "packing" within the fibrous layer, dramatically decreases distinguishability of individual fibers. Thus, images with higher magnifications are more appropriate for the dense layers. As shown from Figure 2 the densest fibrous layers obtained had the narrowest distribution peaks of orientations at 500x magnifications. Conversely, when analysing the images with a magnification of 5000x, a significant change 
towards polydispersion of orientation distribution was observed. Furthermore, one should also consider the fact, that Orientation $\mathrm{J}$ cannot "separate" two overlapping fibers. This drawback has a significant effect on estimated orientation distribution when the magnification of the image is too high and low number of fibers is presented on the view field.

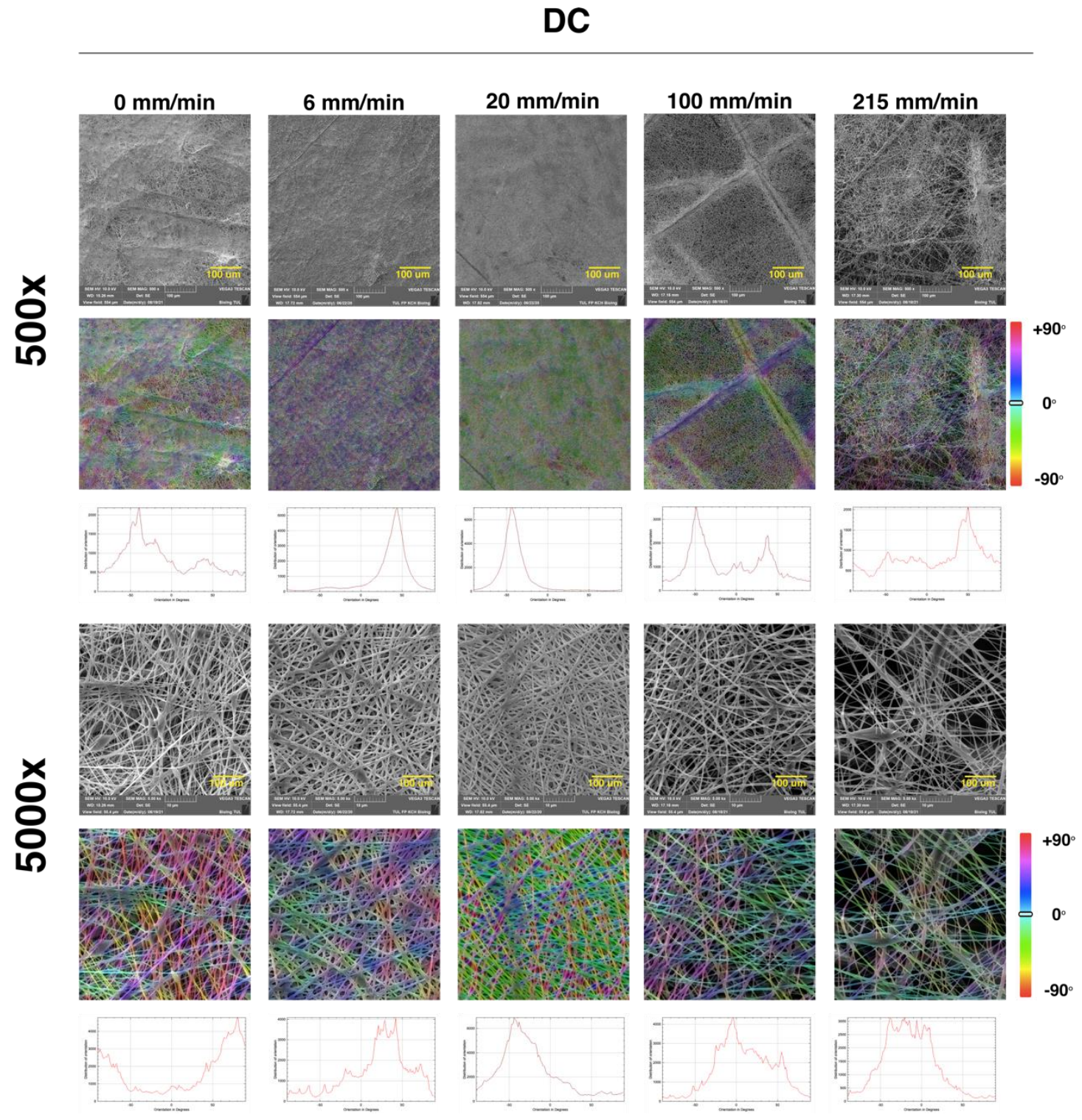

Figure 2 SEM images of fabricated DC electrospun nanofibrous layers and corresponding color-coded analyzed image with histogram of distribution of fiber orientations within the layers

Analysis of the SEM images of AC electrospun fibrous layers revealed that in contrast to DC electrospun layers the orientation was mainly anisotropic at both high and low rewinding speeds. The lowest rewinding speed (30 $\mathrm{mm} / \mathrm{min}$ ) was comparable to the higher speeds (80 and $100 \mathrm{~mm} / \mathrm{min}$ ). This can be partially explained by the nature of AC-spinning process, where the first collected fibers on a collector generally control the orientation of the following fibers. The sample rewound at $80 \mathrm{~mm} / \mathrm{min}$ and left on the roller afterwards to completely solidify had the highest heterogeneity among the AC-spun materials. This is very interesting and shows changes in the fibrous structure when it is dried in the off state. Apparently, certain types of stress transfer occur, and this changes the arrangement of the fibers in the sample with respect to their orientation. This effect should be further studied and explained in detail. Comparing to the SEM images of DC-spun materials, it can be seen from Figure 3, that the apparent isotropy of the layers was increasing with increasing the magnification. This 
is mainly due to the drawback of Orientation $\mathrm{J}$ described above when dealing with a relatively small number of fibers having a large number of intersections, Orientation $\mathrm{J}$ cannot consider the "bottom" fibers as a whole and analysis the fiber as a set of separated segments, thus greatly distorting the resulting distribution of orientations.
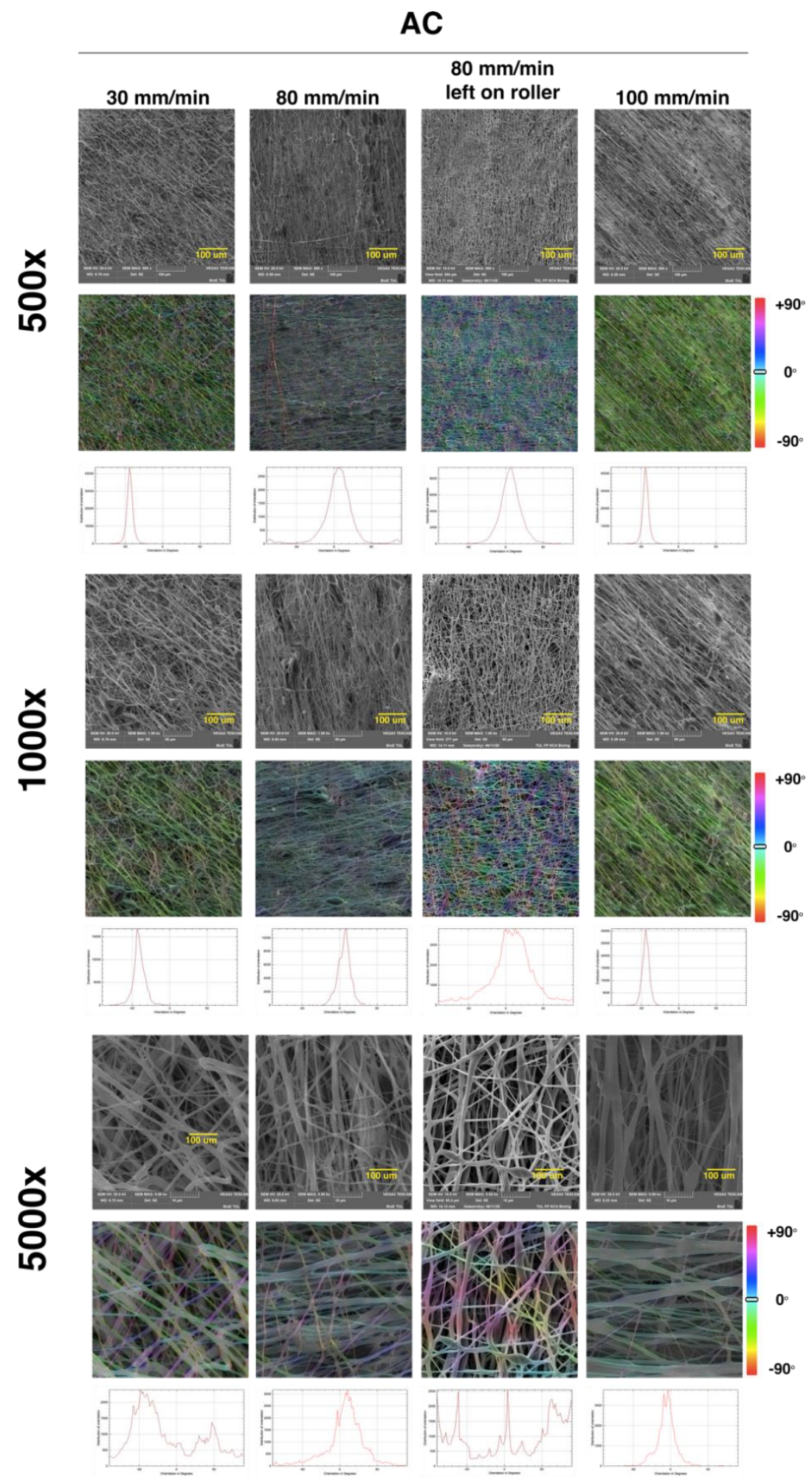

Figure 3 SEM images (different magnifications) of fabricated DC electrospun nanofibrous layers and corresponding color-coded analysed image with histogram of distribution of fiber orientations within the layers 


\section{CONCLUSION}

The orientation distributions of the materials obtained by $A C$ and $D C$ electrospinning differed significantly from each other. The AC fibers had a well-defined prevailing fiber orientation in their majority, while the DC fibers were inherently isotropic at all rewinding speeds. For materials fabricated by DC-spinning, which were characterized by more dense fibrous layers, images at a magnification of $1000 \mathrm{x}$ or higher were more suitable for analysis. On the other hand, in the case of AC-spun fibers, high magnifications distorted the analysis because poorly distinguishable fibers with significantly different $\sigma$ - parameter (structure of interest size) came into play. However, it was shown that at high speeds of material rewinding, a higher level of fiber orientation is achieved, preserving monodisperse decomposition histograms even at high magnifications of the images. Conversely, keeping the fibrous layers on the collector may lead to fiber relaxation, which in turn results in higher isotropy. The established parameters of the measurement could be helpful for the further design of alignment-dependent electrospun materials.

\section{ACKNOWLEDGEMENTS}

This work was supported by the Czech Health Research Council, project No NU20-02-00368.

\section{REFERENCES}

[1] JAHANGIRI, A., ADIBKIA K. Applications of electrospinning/electrospraying in drug delivery. Bioimpacts. [online]. 2016, vol. 6, no. 1, pp. 1-2. Available from: https://doi.org/10.15171/bi.2016.08.

[2] RAHMATI, M. et al. Electrospinning for tissue engineering applications. Progress in Materials Science. [online]. 2021, vol. 117, p. 100721. Available from: https://doi.org/10.1016/i.pmatsci.2020.100721.

[3] BAI, S., ZHANG, X., ZANG, L., YANG, S., CHEN, X., YUAN, A X. Electrospinning of Biomaterials for Vascular Regeneration. Chem. Res. Chin. Univ. [online]. 2021, vol. 37, no. 3, pp. 394-403. Available from: https://doi.org/10.1007/s40242-021-1125-3.

[4] Oriented nanofibrous membranes for tissue engineering applications_Electrospinning with secondary field control. Elsevier Enhanced Reader. [online]. https://reader.elsevier.com/reader/sd/pii/S1751616115002325?token=1DB51A87DF92D115AAAEBF43E6DCAC D56EB773CFEFA662D4CCF34C9CAE43C865EC1DE84864C796ABAC5229083080541A\&originRegion=euwest-1\&originCreation=20210920134909 (Accessed 20 September 2021).

[5] ABUDULA, T., SAEED, U., N. SALAH, N., A. MEMIC, A., H. AL-TURAIF, H. Study of Electrospinning Parameters and Collection Methods on Size Distribution and Orientation of PLA/PBS Hybrid Fiber Using Digital Image Processing. Journal of Nanoscience and Nanotechnology. [online]. 2018, vol. 18, no. 12, pp. 8240-8251. Available from: https://doi.org/10.1166/jnn.2018.15885.

[6] CHIVERTON, J. P., KAO, A., ROLDO, M., TOZZI, A G. Automatic diameter and orientation distribution determination of fibrous materials in micro X-ray CT imaging data. Journal of Microscopy. [online]. 2018, vol. 272, no. 3, pp. 180-195. Available from: https://doi.org/10.1111//jmi.12719.

[7] WALSER, J., FERGUSON, A S. J. Oriented nanofibrous membranes for tissue engineering applications: Electrospinning with secondary field control. Journal of the Mechanical Behavior of Biomedical Materials. [online]. 2016, vol. 58, pp. 188-198. Available from: https://doi.org/10.1016/j.jmbbm.2015.06.027.

[8] ZHANG, L., YU, A W. Orientation image analysis of electrospun submicro-fibers based on Hough transform and Regionprops function. Textile Research Journal. [online]. 2017, vol. 87, no. 18, pp. 2263-2274. Available from: https://doi.org/10.1177/0040517516669070.

[9] POKORNY P., et al. Effective AC needleless and collectorless electrospinning for yarn production. Phys. Chem. Chem. Phys. [online]. 2014, vol. 16, no. 48, pp. 26816-26822. Available from: https://doi.org/10.1039/C4CP04346D.

[10] VALTERA J., et al. Fabrication of dual-functional composite yarns with a nanofibrous envelope using high throughput AC needleless and collectorless electrospinning. Sci Rep. [online]. 2019, vol. 9, no. 1, p. 1801. Available from: https://doi.org/10.1038/s41598-019-38557-z. 